Supporting Information:

\title{
Proximity labeling reveals molecular determinants of FGFR4 endosomal transport
}

Ellen Margrethe Haugsten ${ }^{1,2}$, Vigdis Sørensen ${ }^{1,2}$, Michaela Kunova Bosakova ${ }^{3}$, Gustavo Antonio de Souza ${ }^{4,5}$, Pavel Krejci, ${ }^{3,6}$, Antoni Wiedlocha ${ }^{1,2}$ and Jørgen Wesche $e^{1,2, *}$

${ }^{1}$ Department of Molecular Cell Biology, Institute for Cancer Research, The Norwegian Radium Hospital, Oslo University Hospital, Montebello, 0379 Oslo, Norway

${ }^{2}$ Centre for Cancer Biomedicine, Faculty of Medicine, University of Oslo, Montebello, 0379 Oslo, Norway

${ }^{3}$ Department of Biology, Faculty of Medicine, Masaryk University, Kamenice 5, 625 00, Brno-Bohunice, Czech Republic

${ }^{4}$ Dept. of Immunology, Oslo University Hospital - Rikshospitalet and University of Oslo, 0027, Oslo, Norway

${ }^{5}$ The Brain Institute, Universidade Federal do Rio Grande do Norte, UFRN, Natal-RN, Brazil ${ }^{6}$ International Clinical Research Center, St. Anne's University Hospital, Brno, Czech Republic

Table of contents:

Table legend for supplementary Excel data file (Table 1).

Figure S1.

Figure S2.

Figure S3.

Figure S4. 
Table S1. Analysis of proteomic data. The proteomic data was analyzed and sorted as described in Experimental Procedures. The following samples were analyzed and compared: C1; U2OS-R4 cells (control), C2; U2OS-R4 cells expressing BirA* (control) C3; U2OS-R4 cells expressing BirA* stimulated with FGF1 (control), S1; U2OS-R4-BirA* cells and S2; U2OS-R4-BirA* cells stimulated with FGF1. C3 hits were considered as proteins with induced expression upon FGF stimulation (see "FGF induced expression") and these were removed from the final list. Proteins that are significantly $(p<0.05)$ three-fold or more overrepresented in cells stimulated with FGF1 (S2) when compared to unstimulated cells expressing R4-BirA* (S1) are indicated in red in the final list. These are also presented in Figure 2. 


\section{Figure S1}

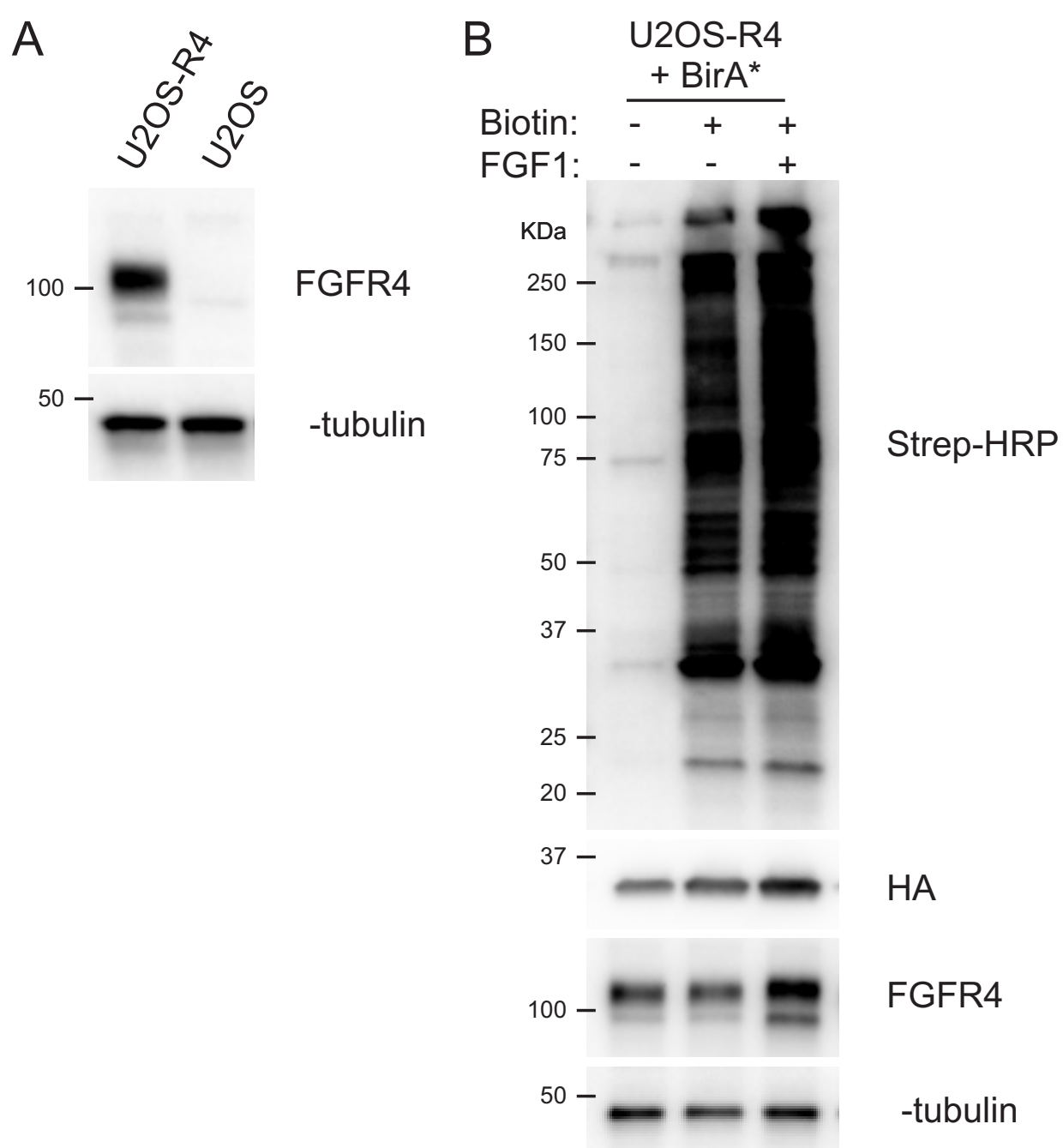

Figure S1. (A) U2OS-R4 cells and U2OS cells were lysed and the cell lysates were analyzed by SDS-PAGE and western blotting using the indicated antibodies. (B) U2OS-R4 cells stably expressing BirA* were treated as indicated for 24 hours and then the cells were lysed and the cellular material was analyzed by SDS-PAGE and western blotting using Streptavidin-HRP (Strep-HRP) and indicated antibodies. 


\section{Figure S2}

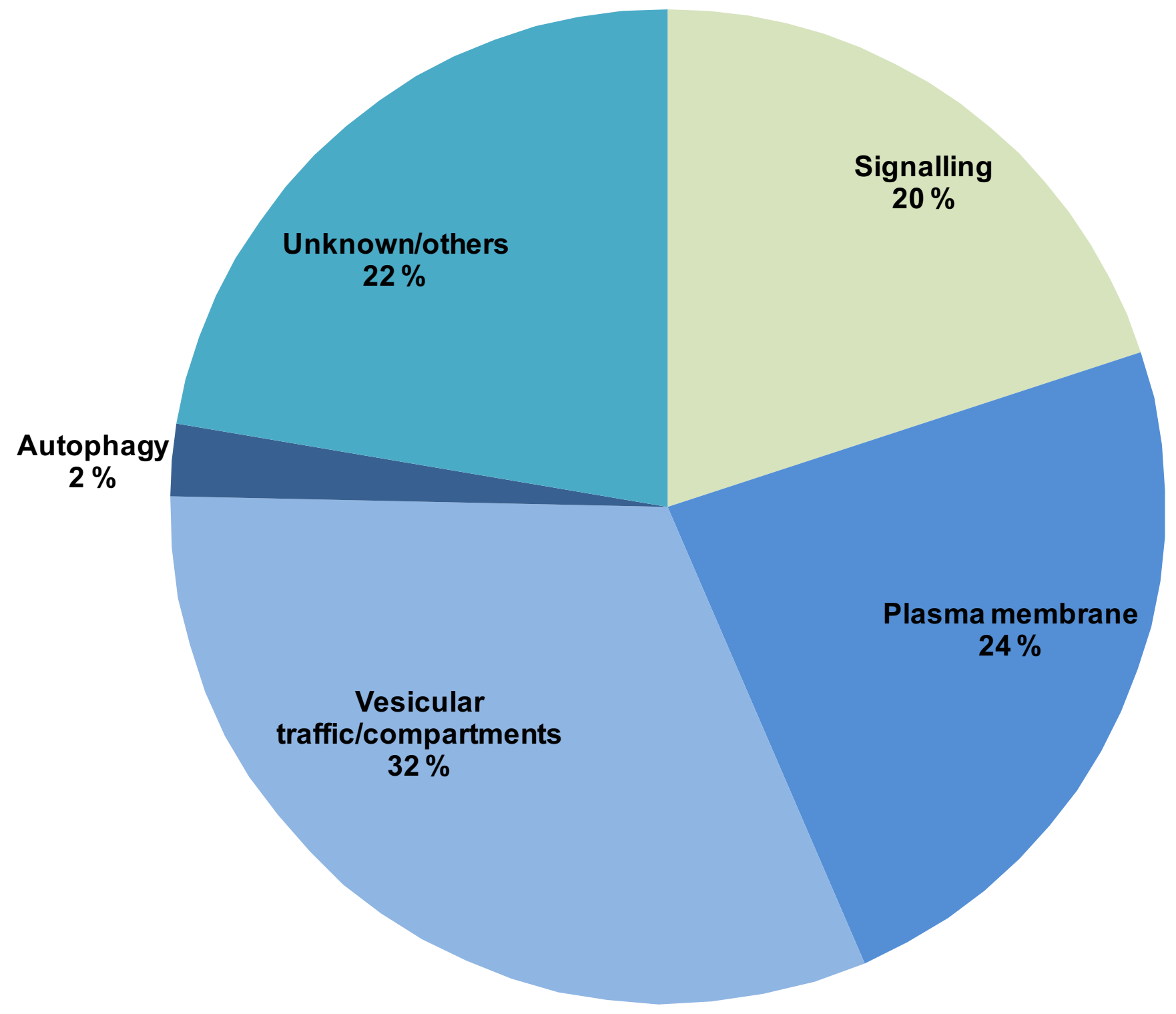

Figure S2. Pie-chart illustrating the classification of proteins as percentage of total proteins according to their function/localization. Information from GeneCards, GO Consortium and literature curation (PubMed) were used to classify the proteins enriched in FGF1 stimulated samples identified by BioID. The proteins were grouped into indicated categories. Some hits mapped to several categories and are therefore included in several categories in the chart. 


\section{Figure S3}

A

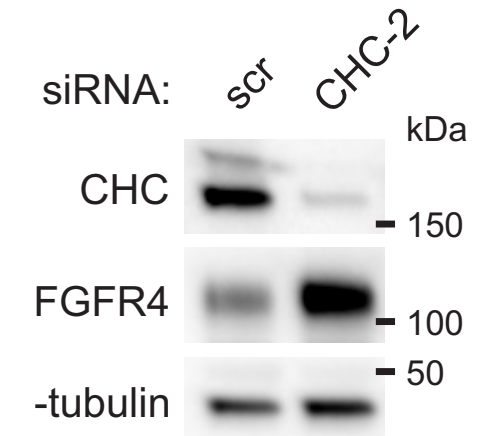

B

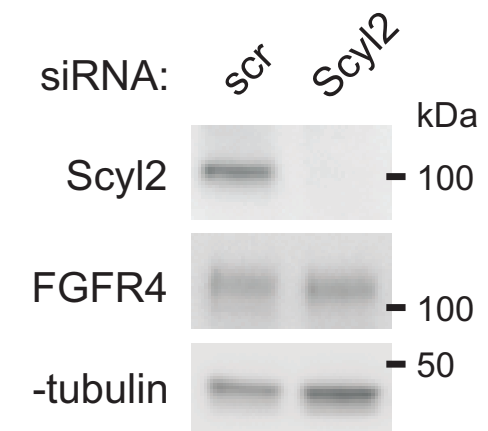

Figure S3. siRNA knockdown efficiencies. U2OS-R4 cells were transfected with non-targeting (scr) or clathrin heavy chain oligo 2 (CHC-2) (A) or Scyl2 (B) siRNA oligos. Seventy-two hours after transfection, the cells were lysed and the cell lysates were then analyzed by SDS-PAGE and western blotting using the indicated antibodies. 
$-\mathrm{scr}$

- CHC-1 siRNA
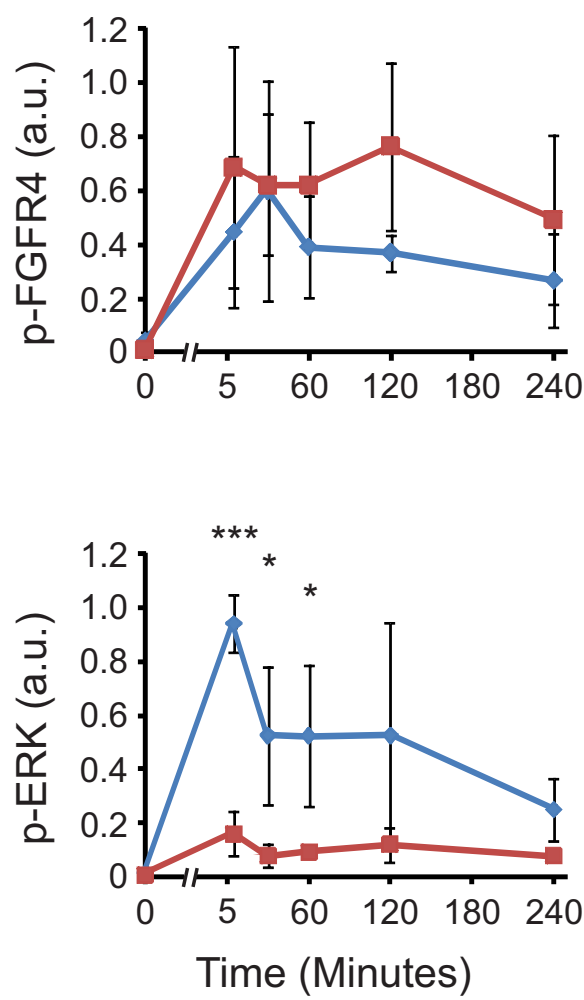
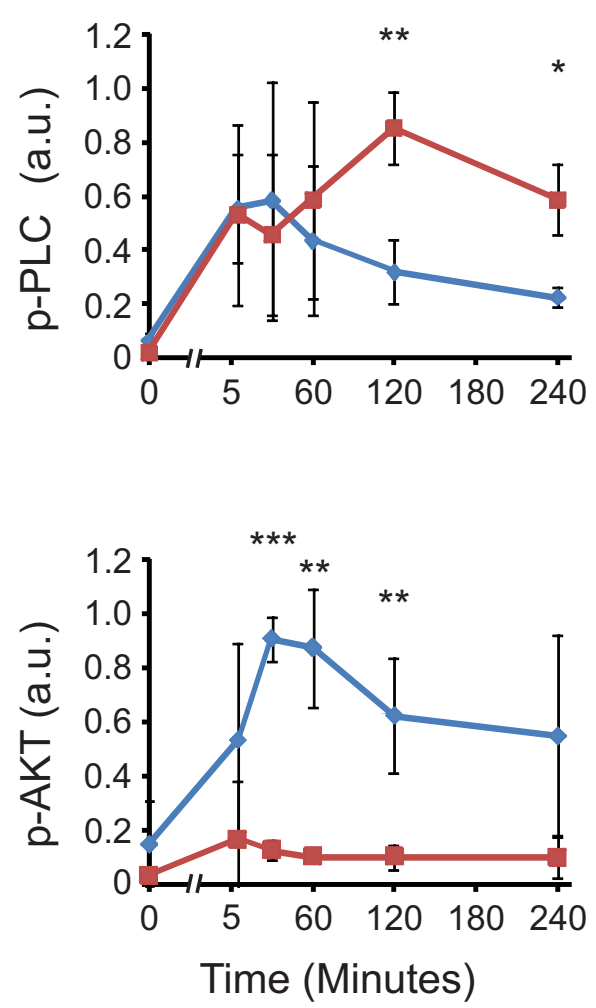

Figure S4. Consequences of clathrin-depletion on FGFR4 signalling with normalization to FGFR4 levels. Western blots as described in Figure 7 were quantified and the bands corresponding to the indicated proteins were normalized to FGFR4 and presented as fraction of maximal response. The graph represents the mean \pm SD of three independent experiments. ${ }^{* * *} p=0.01,{ }^{* *} p=0.01,{ }^{*} p=0.05$. 